\title{
EL CONFLICTO COMO VÍNCULO ENTRE EL GRADO DE ESTRUCTURACIÓN DE PROBLEMAS Y EL ÉXITO DE LA IMPLEMENTACIÓN DE POLÍTICAS
}

Gustavo Valdivieso*

\section{Resumen}

Por más de cuarenta años, la investigación de implementación ha intentado explicar por qué las políticas públicas raras veces alcanzan lo que se espera de ellas. Demasiadas variables relevantes han sido identificadas por los investigadores, haciendo casi imposible realizar predicciones basadas en una, o unas pocas de ellas. Es posible, sin embargo, enfocarse en una versión más reducida del concepto implementación': no lo que se alcanza (los resultados de la política, que son en gran parte determinados por eventos exógenos a la forma en que esta se implementa) sino cómo se alcanza. El modelo aquí planteado propone mirar lo que ocurre con el ritmo de la implementación y la variación en la intervención, y la forma en que son influenciados por la interface entre ambigüedad de la política y grado de estructuración de los problemas. El conflicto es presentado como variable interviniente, y se afirma que es posible realizar predicciones generales sobre los resultados, en términos de ritmo y variación en contextos interorganizacionales, para diferentes combinaciones de nivel de estructuración y ambigüedad de políticas.

Palabras clave: ambigüedad de políticas, estructura del problema, conflicto, implementación, contextos interorganizacionales.

* Doctorante en Estudios Políticos, Universidad Externado de Colombia, Bogotá (Colombia); PhD Researcher, University of Twente, Enschede (Países Bajos). gustavo.valdivieso@uexternado.edu.co

Recibido: 22 de agosto de 2014 / Modificado: 6 de noviembre de 2014 / Aceptado: 21 de enero de 2015.

Para citar este artículo

Valdivieso, G. (2014). El conflicto como vínculo entre el grado de estructuración de problemas y el éxito de la implementación de políticas. OPERA, 16, pp. 131-150. DOI: 10.18601/16578651.n16.08 


\section{CONFLICT AS A LINK BETWEEN PROBLEM STRUCTUREDNESS AND POLICY SUCCESS}

\section{Abstract}

For over forty years, implementation research has struggled to explain why public policy seldom achieves what we expect from it. Too many relevant variables have been identified by researchers, making it almost impossible to make predictions based upon one, or a few of them. It is possible, however, to focus on a smaller version of the concept of implementation: not what is achieved (the policy results, which are largely determined by events exogenous to the way it is implemented) but how it is achieved. The model proposed here propounds looking at pace and variation, and how they are influenced by the interface between policy ambiguity and problem structuredness. Conflict is presented as a mediator between dependent and independent variables, and it is stated that general predictions are possible for pace and variation in inter-organizational implementation settings, for different combinations of problem structuredness and policy ambiguity.

Key words: Policy ambiguity, problem structuredness, conflict, implementation, inter-organizational settings.

\section{INTRODUCCIÓN ${ }^{1}$}

Por más de cuarenta años, la literatura de investigación de la implementación (Implementation Research) ha intentado responder una sencilla pregunta: ¿cómo podemos lograr lo que planificamos en términos de políticas públicas? $\mathrm{O}$, tal vez más exactamente en cuanto al tono: ¿por qué no podemos lograr lo que planificamos en políticas públicas? No es accidental que el subtítulo de la Implementación de Pressman y Wildavsky (1973) rece "Cómo grandes expectativas en Washington se vuelven trizas en Oakland".

Hemos argumentado antes (Valdivieso, 2013) que el grado de estructuración de los problemas no fue tan relevante como debió haber sido en la literatura temprana sobre implementación porque, aunque las literaturas sobre implementación y sobre estructuración de problemas comenzaron a desarrollarse aproximadamente al mismo tiempo hacia 1973, la primera estaba inicialmente sustentada en la aproximación secuencial al proceso de políticas públicas, y se concentró en estudiar programas para los que se suponía que el "problema” estaba dado (y eso es precisamente lo que cuestiona la literatura sobre problemas).

Las discusiones sobre el "problema del problema equivocado" (Raiffa, 1968; Dunn, 1988, 2008), pero también los problemas mal estructurados de Simon (1973) e irresolubles

1 Versiones anteriores de este artículo se beneficiaron de comentarios y sugerencias de los profesores Robert Hoppe y Richard Matland, así como de largas conversaciones con el profesor Gonzalo Ordóñez. 
(wicked) de Rittel y Weber (1973), son abordadas en ciertas ocasiones al tratar el diseño, pero no la implementación. En el clásico de Pressman y Wildavsky, por otra parte, la complejidad de la acción conjunta se explica desde la multiplicidad de puntos de coordinación, sin mayor relevancia para el problema de las diferencias de los actores sobre la naturaleza del problema.

Puede haber otras razones para la poca atención prestada a la estructuración del problema: desde la tendencia a considerarla consecuencia natural de la ambigüedad, entendida a su vez como resultado natural de la necesidad de negociar las políticas (por ejemplo, Birkland, 2011), hasta cuestionar la necesidad misma de la estructuración de problemas, a partir de la posición de Lindblom (1959) que cuestiona todo el análisis "racional".

Las teorías social-constructivistas han dado una nueva oportunidad al grado de estructuración de los problemas, dado que el corazón mismo del constructivismo es la idea de representaciones socialmente construidas de la realidad —esto es, en análisis de políticas, de los problemas-. El "problema del problema equivocado" ya no es uno de diagnosis que podría ser "resuelto" vía mejores técnicas —aunque por supuesto, el análisis deliberativo de políticas puede ayudar a manejarlo- - Por definición, cada descripción que un grupo hace de un problema corre el riesgo de ser considerada "equivocada" por otro grupo.

Pero falta explicar cómo la estructuración de problemas puede afectar la implementación. Y en qué circunstancias lo hace más o menos. Las siguientes secciones estarán dedicadas, primero, a explicar cómo entendemos las diferentes variables que interactúan en el modelo, y, luego, a explicar la forma en que funcionan dentro de él.

\section{EL ROL DEL CONFLICTO}

El modelo que someteremos a prueba durante el proceso de esta investigación postula que el vínculo entre el grado de estructuración de los problemas (entendido como grado de consenso sobre los objetivos que se buscan y los medios susceptibles de ser utilizados) y la implementación reside en la variable interviniente "conflicto", y que el nivel de estructuración de los problemas influencia el éxito de la implementación vía la generación —o no- de conflicto.

Más concretamente, afirmamos que dos variables independientes (nivel de estructuración del problema y ambigüedad de la política) actúan sobre una variable interviniente (conflicto) que tiene efectos sobre dos variables dependientes (ritmo de la implementación y variaciones del proyecto/programa en ejecución). La presencia de conflicto se traducirá en un menor ritmo de implementación y en una mayor variación de lo que se implementa, pero la dimensión de la influencia del conflicto será menor si hablamos de implementaciones que requieren niveles bajos de interdependencia entre actores, y mayor para niveles más altos de interdependencia. La variable que conecta las independientes con las dependientes es la variable interviniente o mediador: conflicto.

¿Cómo definimos conflicto? Richard Matland (1995), de quien tomamos el concepto, no lo define, pero se refiere a "disputas" acerca de objetivos o medios de políticas que 
pueden escalar "con un incremento en la incompatibilidad de las preocupaciones, y con un incremento en el monto percibido de lo que tiene en juego cada actor. Mientras más importante sea la decisión, más agresiva será la conducta” (p. 156).

Citando a Dahrendorf (1958), Matland afirma que "para que exista conflicto debe existir una interdependencia de actores, una incompatibilidad de objetivos, y una percepción de suma-cero en la interacción" (1995, p. 156). Para él, lo más importante sobre el conflicto es la forma en que influencia la selección de un estilo apropiado para la implementación (administrativa, política, experimental, simbólica) que pueda ayudar a resolver el conflicto o al menos disminuir su impacto.

El trabajo del profesor Matland llamó la atención sobre el conflicto al destacar sus posibles efectos benéficos sobre la implementación en algunas circunstancias, así como las diferentes implicaciones que tiene para escoger las estrategias de implementación ideales cuando se combina con distintos niveles de ambigüedad de la política.

Para nuestro modelo, las disputas asociadas con conflicto pueden asimilarse a frenos autoimpuestos a la cooperación, no necesariamente contemplando la posibilidad de confrontación abierta o conducta explícitamente agresiva de una parte hacia otra(s). Lo que es más interesante sobre el conflicto, en este caso, no es la mejor manera de resolverlo, sino la magnitud de su influencia sobre el ritmo de la implementación y la variación en las políticas o los programas. Es posible afirmar que el conflicto es solucionable y, al mismo tiempo, que resuelto o no, su existencia tendrá implicaciones en términos del ritmo y la variación en las actividades (normas, programas, proyectos) por implementar.

\section{EL CONTEXTO: IMPLEMENTACIÓN INTERORGANIZACIONAL}

Es útil presentar aquí los conceptos por discutir, comenzando por el principio: a qué nos referimos con "implementación": nos referimos al cumplimiento de las actividades que implica una política.

Nos enfocamos en políticas específicas, siguiendo la definición que hace Matland de políticas como "las actividades programáticas formuladas en respuesta a una decisión dotada de autoridad”. La atención está, no como en la aproximación de Dye (1987) en "cualquier cosa que el Gobierno decida hacer o no hacer" en un campo de política dado, sino en la implementación de actividades concebidas para ejecutar una decisión dotada de autoridad en ese campo (decisión que normalmente es un programa, un proyecto o una norma).

A diferencia de Matland, sin embargo (y a diferencia de muchos otros), este modelo define la implementación exitosa, no como el logro de objetivos —aun cuando estén "claramente formulados" y puedan tener legitimidad democrática-, sino como la realización a tiempo de las tareas y los productos programados para alcanzar esas metas, con relativamente baja variación programática en entornos específicos durante el proceso.

¿Por qué no definir la implementación en términos de logros? O’Toole (2000) definió la implementación como "lo que ocurre entre el establecimiento de una aparente intención por 
parte del Gobierno de hacer algo, o de dejar de hacer algo, y el impacto final en el mundo de la acción”. Mazmanian y Sabatier (1989) habían sido más ambiciosos, incluyendo "la aprobación del 'estatuto básico', las decisiones de las agencias implementadoras, el cumplimiento por los grupos objetivo y los impactos reales - tanto intencionales como no- de esos resultados, los impactos percibidos de las decisiones de las agencias, y finalmente, revisiones importantes (o revisiones intentadas) al estatuto básico". Pero, como lo resaltaron van Meter y van Horn (1975), los objetivos pueden o no ser alcanzados, independientemente de la calidad de la implementación. De acuerdo con el "marco de coaliciones de abogacía" de Sabatier y Jenkins-Smith (1993), por otra parte, una política puede evolucionar manteniendo sus elementos principales, mientras modifica otros, a lo largo de un periodo de varios ańos, al menos parcialmente como resultado del aprendizaje sobre políticas - aunque también con la influencia decisiva de cambios en las coaliciones de abogacía-.

Si la evaluación de resultados solo es adecuada después de algunos años de aprendizaje sobre las políticas, esto implica que los logros de políticas solo deberían medirse tras ese sustancial periodo de tiempo. Pero cada "versión" de la política a lo largo de ese periodo puede ser identificada en los diferentes programas diseñados para ejecutarla, y cada uno de esos programas acopla un conjunto de actividades que se espera desarrollar.

Las actividades ocurren en periodos mucho más cortos que los logros, y podemos saber mucho más pronto si se están desarrollando de acuerdo con el plan. Así es como funciona el monitoreo de programas: podemos saber si las madres están siendo efectivamente entrenadas en la preparación adecuada de alimentos (actividad) mucho antes de que podamos establecer si la desnutrición infantil está disminuyendo (logro), y más aún, si está cediendo como consecuencia de nuestra intervención.

Contemplar la implementación a través del cumplimiento y la variación de tareas, en vez de hacerlo mediante el logro de objetivos, es más adecuado para el estudio de programas específicos, y demanda horizontes de tiempo menores, haciendo el análisis causal y la modelación más útiles en comparación con estudios longitudinales que son más útiles para estudiar los logros, y en los cuales técnicas como el process tracing están mejor dotadas para explicar evoluciones de largo plazo donde múltiples variables pueden cambiar su influencia sobre los resultados en momentos diferentes de los procesos.

Ahora, nos referimos concretamente a implementación multiactores: esta es la que ocurre en contextos interorganizacionales, donde siempre hay algún nivel de interacción entre actores tratando de ejecutar una política pública.

Si tomamos a la organización como la unidad de análisis, un programa que involucra, por ejemplo, el entrenamiento de técnicos por una agencia del Gobierno o la construcción de caminos por una municipalidad, puede plausiblemente realizarse con la participación de un solo actor (esa organización) incluso si implica contratar varios contratistas individuales. 
Es cierto, sin embargo, que una parte grande —y quizá creciente — de toda la implementación de políticas se realiza involucrando múltiples actores (organizaciones) y generando algún nivel de interdependencia entre ellos. En Colombia, políticas en temas como generación de ingresos o atención a víctimas son ejecutadas por múltiples actores. Incluso normas muy concretas (regulación de locales nocturnos) o proyectos específicos (formalización de vendedores ambulantes) son ejecutados por varias organizaciones con algún nivel de interdependencia entre ellas. Entre las razones detrás del surgimiento de la implementación interorganizacional, O’Toole (2007) identifica la expansión de la agenda gubernamental, demandas crecientes para el abordaje por los gobiernos de temas "que no pueden categorizarse limpiamente en uno $\mathrm{u}$ otro nicho" y las fuerzas de la globalización, "en particular el ímpetu para el trabajo conjunto internacional que emerge de la adopción de acuerdos internacionales", frecuentemente requiriendo acciones con la participación de diferentes ministerios, gobiernos y sectores dentro de un país (p. 236).

Cuando tomamos la organización como unidad de análisis respecto a la implementación, la diferencia fundamental entre la implementación organizacional y la interorganizacional se encuentra en la aplicabilidad de la autoridad para garantizar la coordinación. Según el mismo O’Toole (2007), el principio en el que se enmarca gran parte de la asesoría en administración es que los gerentes pueden esperar tener una habilidad significativa para dar órdenes y direccionar recursos en cuanto se refiere a la parte central de sus dominios. Pero en un mundo de relaciones interorganizacionales, esa premisa es cuestionable:

La jerarquía formal permite a los administradores públicos en departamentos depender en un grado considerable en la autoridad como ayuda para la coordinación. Pero los administradores que trabajan más allá de esas fronteras generalmente no poseen ese privilegio. Puede haber puntos formales de autoridad entre diferentes departamentos - la oficina del ejecutivo principal, por ejemplo, o el Gabinete- pero en la práctica esa autoridad casi nunca es invocada. Los oficiales centrales tienen poco que ganar dejándose arrastrar a las disputas ministeriales y típicamente tienen la expectativa de que las organizaciones resuelvan sus diferencias. E incluso el tiempo y la autoridad de los decisores centrales son estrictamente limitados y usualmente racionados para los casos más urgentes (p. 237).

Gran parte de la relevancia de la estructuración de problemas para el estudio de la implementación de políticas públicas se explica por la posibilidad teórica de que pueda sustituir a la autoridad como soporte para la cooperación en ambientes interorganizacionales. Lo que algunos autores llaman "discreción burocrática” (por ejemplo, Thompson, 1982) se incrementa en ambientes interorganizacionales, con menos restricciones para la acción impuestas desde el centro. Niveles altos de estructuración del problema, entendida como consenso sobre las dos dimensiones del problema (objetivos y medios susceptibles de ser usados) traen consigo mejores prospectos para la cooperación y la coordinación en presencia de discreción significativa. 
LAS VARIABLES INDEPENDIENTES: NIVEL DE ESTRUCTURACIÓN DEL PROBLEMA, AMBIGÜEDAD DE LA POLÍTICA E
INTERDEPENDENCIA REQUERIDA

Las variables independientes en este modelo son: estructuración de los problemas, ambigüedad de la política y nivel de interdependencia requerida, con conflicto como la variable interviniente influenciada por la interface entre ambigüedad y estructuración, que interactúa a su vez con la interdependencia requerida para influenciar las variables dependientes ritmo de implementación y variación.

La estructuración de problemas es un concepto desarrollado en la literatura de investigación de operaciones/análisis de políticas en los ańos sesenta y setenta, cuando se hizo evidente la extrema dificultad de hallar soluciones "óptimas" para muchos problemas de política pública. Referencias relevantes de esa época son la exploración de "problemas mal estructurados" de Simon (1973), el estudio de "problemas irresolubles" (wicked) de Rittel y Weber (1973), y las reflexiones de Ackoff sobre "revoltijos" (messes) (1974). Otras contribuciones significativas desde la perspectiva del análisis de políticas incluyen a Dunn (1988, 2008), y, por supuesto, hay una rica literatura que sigue creciendo en investigación de operaciones y una serie de métodos de estructuración de problemas (PSM por su sigla en inglés) referenciados en ella.

Mingers y Rosenhead (2009), citando a Checkland, describen los problemas de los que se ocupa la investigación de operaciones a través de los siguientes componentes: a) una situación inicial (So), b) una situación deseada
(S1) y c) un conjunto de alternativas para pasar de So a S1. "La resolución de problemas, según esta perspectiva, consiste en definir So y S1 y seleccionar los mejores medios para reducir la diferencia entre ellas" (pp. 6-7). Así, inferimos, mientras mayor incertidumbre exista sobre cuáles son los estados inicial y deseado, cuál es el conjunto de alternativas y cuáles los criterios relevantes para escoger "la mejor", menos estructurado estará el problema.

Si hay algo de consenso sobre lo que un problema estructurado es, hay menos consenso sobre las condiciones que permiten tener un problema estructurado. Simon (1973) argumentó que un problema está bien o mal estructurado dependiendo de la cantidad de información que se aporte al tomador de decisiones (con mayores cantidades de información dificultando frecuentemente la decisión), y por tanto, ningún problema está intrínsecamente bien o mal estructurado. Al describir los wicked problems (problemas irresolubles) Rittel y Weber incorporaron, además de la falta de una formulación definitiva y la carencia de una "regla de pare" (stopping rule) —una regla para identificar que una solución ha sido alcanzada - , la falta de un conjunto definido de potenciales soluciones y la multiplicidad de formas en que pueden ser entendidos como características de estos.

Dunn (1988) subraya que en los problemas mal estructurados: a) los tomadores de decisiones, b) las preferencias o utilidades, c) los resultados y d) los estados actuales de la naturaleza son desconocidos o inciertos. La razón para esas dificultades es la multitud de actores involucrados, dado que continuamente se crean, mantienen y modifican "representa- 
ciones conflictivas por actores involucrados que afectan, y son afectados por, los procesos de construcción de política de los gobiernos modernos" (p. 722).

Ciertamente, más actores interesados y más alternativas están frecuentemente asociados con menos consenso y problemas menos estructurados, pero para nuestro modelo hemos escogido la aproximación de Robert Hoppe (2010), donde el foco no está en el número de actores o de alternativas, o en la posibilidad de predecir el resultado de las alternativas, sino en la existencia o ausencia de consenso en cuanto a los objetivos y los medios plausibles en sí mismos, cualquiera sea el número de actores interesados (o de organizaciones participantes en nuestro caso), y cualquiera el número y la precisión del conocimiento sobre las alternativas.

Además, el modelo de Hoppe da a la estructuración respecto a medios igual importancia que a la estructuración respecto a fines. Eso no es el caso en Dunn, y ciertamente no lo es en la mayoría de la más bien escasa literatura que aborda el "conflicto" en la toma de decisiones, que se centra en el conflicto sobre objetivos y destaca por esa razón las preocupaciones sobre "claridad de objetivos" (goal clarity) e incluso claridad de mandato (mandate clarity) que son tan propias de las aproximaciones top-down (por ejemplo, Mazmanian y Sabatier, 1989; May, 1993). Lindblom y Pressman-Wildavsky se encuentran entre las excepciones, y en ambos casos se resalta un punto más bien diferente: el consenso sobre medios (o sobre subobjetivos) puede ser más difícil de alcanzar que el consenso sobre objetivos.
En la tipología de Hoppe (2010), cada problema público puede estar estructurado, moderadamente estructurado (estructurado respecto a medios), moderadamente estructurado (estructurado respecto a fines) o no estructurado. Un nivel de estructuración es entendido como la situación de un problema público en un determinado momento del tiempo, una situación que aunque dinámica no es necesariamente parte de un proceso (de estructuración de problemas) en el que exista certeza de que el problema estará estructurado al final, si se cumplen ciertas condiciones (figura 1). Esto es, a diferencia de Dunn, Robert Hoppe acepta la posibilidad de que un problema de política pública sea wicked en los términos de Rittel y Weber (1973): básicamente irresoluble, porque es inestructurable, al menos en cierto punto en el tiempo.

\section{FIGURA 1. TIPOLOGÍA DE ESTRUCTURAS DE PROBLEMAS DE ROBERT HOPPE (2010)}

Lejos de la certeza sobre conocimiento requerido y disponible

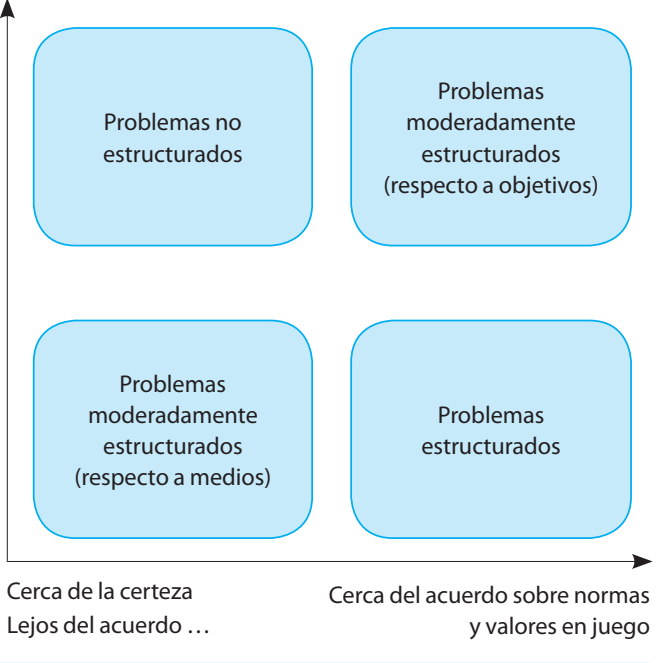

Fuente: adaptado de Hoppe (2010, p. 73). 
Tomando la aproximación social-constructivista de Hoppe a los problemas de política, y entendiéndolos como construcciones cognitivas sociales, asumimos que todo problema público puede ser estructurado, moderadamente estructurado o no estructurado en diferentes momentos del tiempo, dependiendo del mayor o menor nivel de acuerdo sobre lo que el problema es entre los actores involucrados relevantes. Las reformas institucionales y la liberalización de mercados, por ejemplo, eran vistas como la solución a los problemas de la pobreza en América Latina a principios de la década de los noventa, haciendo de ese un problema relativamente bien estructurado, mientras que hoy ese consenso es claramente menor, y podemos decir que el nivel de estructuración del problema es menor.

Hoppe (2010) aporta ejemplos de problemas que pasan de una situación no estructurada a una estructurada (screening prenatal para detectar anomalías en los fetos en Holanda entre 1990 y 2007, donde objeciones éticas combinadas con incertidumbres técnicas fueron sustituidas por un amplio consenso acerca del acceso condicionado a este tipo de escaneo para ciertas categorías de mujeres y asesoría profesional para tomar decisiones bien informadas). Pero también del ejemplo contrario: la contaminación de suelos en el Volgermeer Polder, donde un problema aparentemente estructurado de capacidad fue abandonado en favor de un problema de salud no estructurado, para finalmente estabilizarse como "un problema moderadamente estructurado con algo de consenso sobre objetivos". En estos ejemplos, el cambio de problema estructurado a no estructurado (o viceversa) no depende de las técnicas de análisis utilizadas.

Respecto a la ambigüedad de la políti$c a$, la clasificamos, siguiendo a Matland, en ambigüedad respecto a medios y respecto a objetivos (figura 2). Una de las principales contribuciones de Matland fue no juzgar a la ambigüedad como negativa per se, destacando en cambio su contribución para evitar el conflicto durante el diseño de políticas —un punto en el que muchos académicos están de acuerdo, como vimos arriba - pero también su carácter de "oportunidad para aprender tanto nuevos medios como nuevos objetivos" (1995, pp. 159-167). El modelo de Matland, con su énfasis en los posibles efectos positivos de la ambigüedad, es particularmente útil cuando se estudia uno de los tres posibles escenarios de la implementación multiactor (interdependencia operacional combinada, ver adelante).

"Dividirla" en dos ayuda a operacionalizar la variable ambigüedad (Bouma y Ling, 2004). Se considera que la ambigüedad varía en un continuo entre niveles relativamente bajos y relativamente altos. Un programa con objetivos definidos de forma muy amplia - por ejemplo, reducir la violencia en el departamento de Casanare, Colombia-implica mayor ambigüedad que uno con objetivos más estrechos - por ejemplo, reducir la violencia con armas de fuego en los municipios de Yopal y Tauramena, departamento de Casanare, Colombia- En cuanto a la ambigüedad respecto a medios, es mayor para un programa que no especifica medios sugeridos que para otro que aclara que contenidos mediáticos y demostraciones de fuerza de la Policía son los 
FIGURA 2. EFECTO COMBINADO DE CONFLICTO Y AMBIGÜEDAD DE POLÍTICAS

EN EL MODELO DE MATLAND (1995)

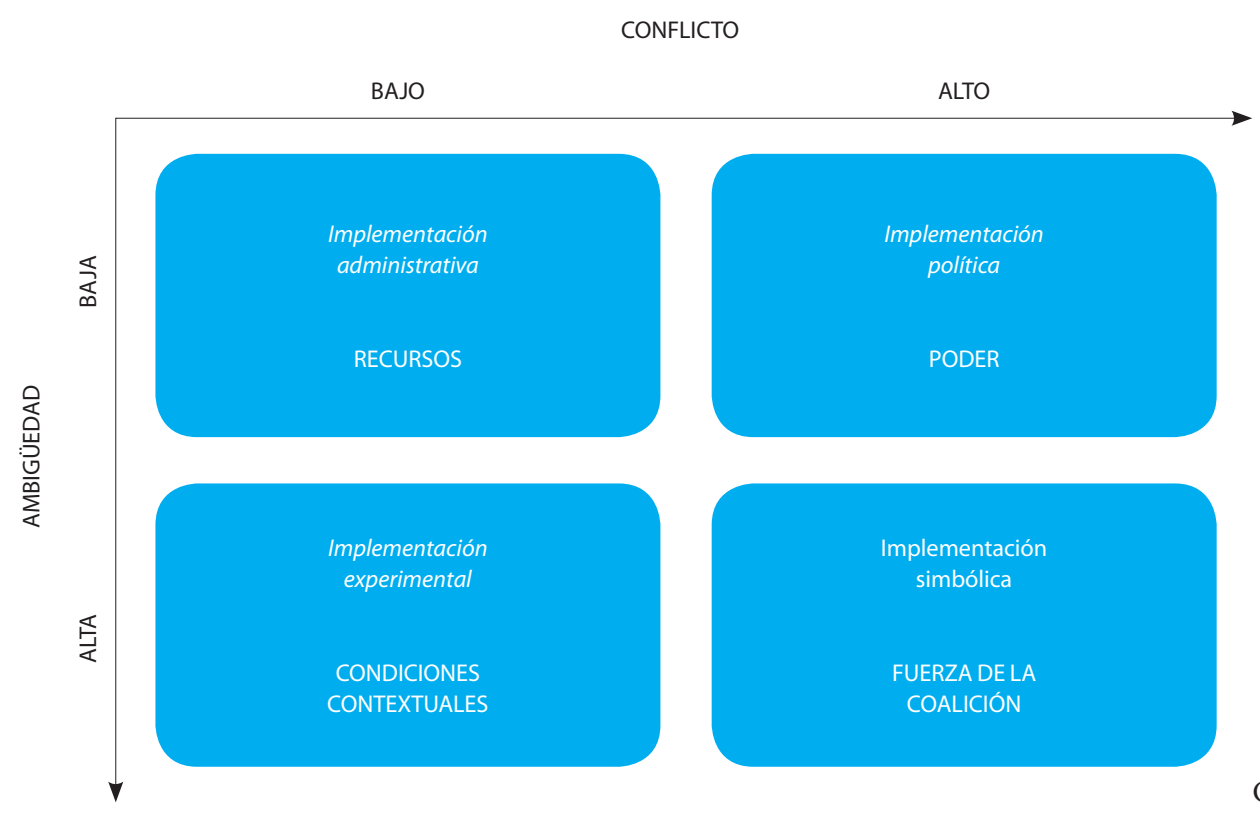

C

Fuente: adaptado de Matland (1995, p. 160).

Nota: los conceptos arriba de cada celda se refieren al tipo de implementación más adecuado para la combinación de ambigüedad y conflicto en cada caso. Los conceptos en la parte inferior de cada celda se refieren al factor que se espera tenga mayor influencia en cada caso.

vehículos apropiados para influenciar la conducta de los residentes en Yopal o Tauramena.

Interdependencia requerida se refiere al nivel de interdependencia esperada entre las diferentes organizaciones según el diseńo de la política. O’Toole y Montjoy (1984), utilizando las categorías de Thompson (1967), identifican tres tipos de interacción - y tres niveles de interdependencia - en la implementación multiactor: a) interdependencia operacional combinada, donde el nivel de coordinación requerida es el mínimo (múltiples agencias operan sobre el mismo objeto durante la implementación, pero ninguna de ellas debe recibir insumos de las demás para hacer su parte del trabajo, aunque la coordinación podría mejorar el resultado; b) interdependencia operacional secuencial, en la cual "un retraso o rompimiento en algún punto afectará las operaciones de las unidades subsecuentes en la cadena de implementación”, y c) interdependencia operacional recíproca, en la que las agencias "deben ajustarse mutuamente para cooperar con las demás”. Los casos b y c son aquellos con mayores niveles de interdependencia requerida (O’Toole y Montjoy, 1984, pp. 493-494). Según este modelo, el conflicto 
tiene diferentes consecuencias para el ritmo y la variación en la implementación en presencia de diferentes tipos de interdependencia.

\section{LAS VARIABLES DEPENDIENTES: RITMO Y VARIACIÓN}

El éxito en la implementación es el constructo conceptual general que planeamos abordar al mirar los efectos de la interface entre nivel de estructuración del problema, la ambigüedad de la política y la interdependencia requerida. Pero dijimos antes que implementación es un concepto demasiado amplio, que incluye "lo que se desarrolla entre el establecimiento de una aparente intención de hacer o dejar de hacer algo por parte del Gobierno y el impacto final en el mundo de la acción", aun en la definición restringida de O'Toole. A falta de mayores precisiones, "lo que se desarrolla" significa exactamente lo mismo que "cualquier cosa que ocurra". Y es imposible medir "cualquier cosa que ocurra" para someter a prueba una hipótesis.

Proponemos no mirar "cualquier cosa que ocurra", ni tampoco "el impacto final sobre el mundo de la acción" como propone O’Toole, por ejemplo. Lo que queda es el estudio de las actividades programáticas per se. La primera pregunta que nos viene a la mente es si esas actividades fueron completadas; la segunda es ¿̇a qué ritmo? Entre decenas de variables dependientes e independientes en la lista de O'Toole (1986), "tiempo" es la más común.

Aquí se escogió una segunda variable propuesta para estudio, dada su relación con la variable interviniente (conflicto). Si los par- ticipantes en un contexto interorganizacional de implementación no logran consensos rápidos sobre qué hacer, sosteniendo distintas visiones acerca de ello, una forma de superar esas diferencias será mediante la negociación, generando modificaciones en el programa/ proyecto original (variación). Pero la variación no solo es un signo de negociación. Puede tener implicaciones en términos de recursos requeridos, resultados alcanzados (aunque ya dijimos que no abordaremos los resultados) y también efectos sobre el ritmo de la implementación (aunque no podemos decir que el ritmo sea una función de la variación con respecto a las actividades inicialmente planificadas).

Así, para hacer operacional el concepto (Bouma y Ling, 2004) definiremos éxito de la implementación en términos de dos variables operacionales: ritmo de la implementación de la decisión (programa, proyecto o norma) y variación en las actividades respecto a las planteadas inicialmente. Para cada una de esas variables operacionales, sin embargo, tendremos que encontrar indicadores apropiados que sean aún más precisos en su significado — y de esa forma, más fácilmente medibles—.

\section{UNIDADES DE ANÁLISIS}

Las actividades programáticas - y las actividades en general- son las unidades de análisis lógicas para el estudio de la implementación. Uno puede mirar la implementación como un "proceso de ensamblaje", referido a construir una máquina a partir de sus piezas y ponerla a funcionar (Bardach, 1977) (una perspectiva muy top-down) o, como "lo que 
ocurre entre el establecimiento de una aparente intención por parte del Gobierno de hacer algo, o de dejar de hacer algo, y el impacto final en el mundo de la acción" (O'Toole, 2000), en una perspectiva más bottom-up en la que no hay necesidad de esa línea de montaje y los actores pueden no estar coordinados en absoluto. Y, sin embargo, en ambos casos la implementación, sea de una decisión tomada en las altas esferas del Gobierno nacional o de una tomada a un nivel muy bajo/local, se trata siempre de realizar actividades en respuesta a una decisión dotada de autoridad (entrenar personas, arrestar personas, recaudar impuestos de personas, irrigar terrenos, patrullar parques). Hay actividades programáticas aun en ausencia de un programa o proyecto formal, siempre que una decisión dotada de autoridad, como una norma, requiera dar ciertos pasos para hacerla cumplir.

Aunque hemos subrayado en otras partes la importancia de un análisis multinivel de la implementación que tome en cuenta, no solo las variables que actúan a nivel de programas, sino al nivel organizacional y de la política (Sandfort, Roll y Moulton, 2013) y, más aún, la relevancia de tomar en cuenta el nivel del modo de gobernanza (Hill y Hupe, 2007; Howlett, 2011), es cierto que toda variable que afecte la implementación debe tener, en última instancia, un impacto en la arena en la que la implementación ocurre finalmente, y esa es la de las actividades programáticas.

En el caso de la investigación específica que nos planteamos para "falsear" este modelo, nuestra unidad de análisis será el proyecto, dado que lo que llamamos en Colombia "programa" es muchas veces diferente del concepto establecido en la literatura y esto puede generar confusiones. En la planificación del Gobierno colombiano, un programa es muchas veces un "área de actividad", no necesariamente implicando direcciones para la acción.

En los proyectos tenemos una unidad de análisis "incrustada” (Yin, 2009): las organizaciones, aquellas que interactúan en contextos interorganizacionales en forma cooperativa (o se abstienen de hacerlo, dependiendo del nivel de estructuración del problema, de acuerdo con nuestro modelo).

\section{MODELO DESCRIPTIVO DE LA RELACIÓN ENTRE EL NIVEL DE ESTRUCTURACIÓN DE LOS PROBLEMAS, LA AMBIGÜEDAD DE LAS POLÍTICAS Y DOS DIMENSIONES ESPECÍFICAS DEL ÉXITO DE LA IMPLEMENTACIÓN}

El primer hecho respecto al modelo es que es descriptivo, un intento de clarificar relaciones entre variables, y no prescriptivo, tratando de sugerir cómo mejorar la implementación mediante la manipulación de variables, aún para las dos variables dependientes específicas escogidas aquí.

Un segundo hecho, es que este modelo busca ayudar a clarificar relaciones entre el nivel de estructuración de problemas, la ambigüedad de la política, el conflicto, el ritmo de implementación y la variación para implementación llevada a cabo en contextos interorganizacionales.

Asumir las condiciones existentes cuando se está en presencia de un solo tomador de 
decisiones fue una de las principales debilidades del análisis de políticas original - lo que Majone (2005, original 1989) llamó decisionismo-, que solo se hizo más evidente cuando la investigación sobre la implementación (por ejemplo, Pressman y Wildavsky, 1973) identificó la existencia no de uno, sino de múltiples puntos de decisión a lo largo del proceso de implementación. Cada uno de esos puntos es una instancia donde deben tomarse decisiones sobre actividades. ¿Deberíamos contratar trabajadores (actividad) de acuerdo con estos criterios, o con algún otro? La diferencia relevante entre la implementación uniactor y multiactor en este aspecto es que en la implementación uniactor la relación entre estructuración de problemas y ritmo de implementación está mediada solamente por el número de variables que el actor identifique como relevantes para el problema y sus capacidades computacionales para decidir entre ellas, esto es, lo que Paul Sabatier llamó la "tractabilidad" del problema, la capacidad de "resolverlo" mediante computación. Una organización implementadora solitaria, como el departamento de construcción municipal al que nos referimos antes, no debería ver su ritmo de implementación o la variación en sus proyectos afectados por necesidades de cooperación.

Si seguimos la tipología de Hoppe, es teóricamente posible tener problemas estructurados en procesos de implementación multiactor, ya que el nivel de estructuración no es una función del número de actores involucrados sino del nivel de acuerdo sobre el problema entre ellos. La literatura sobre implementación interorganizacional (por ejemplo, O’Toole, 2007) afirma que con bastante frecuencia la falta de estructuración entendida de esta manera es más la norma que la excepción.

La afirmación general del modelo, repetimos, es la siguiente: Ceteris paribus, en contextos de implementación interorganizacionales con ambigüedad en la política, programa o proyecto implementado, una mayor estructuración de problemas está asociada con menores niveles de conflicto entre los implementadores, y por esta vía con mayores niveles de éxito en la implementación.

Aunque hay niveles relativamente altos de discreción para los actores (implementadores), dada la ausencia de relaciones jerárquicas entre ellos o la ausencia de voluntad para hacer sentir esas relaciones jerárquicas, la presencia de altos niveles de estructuración de los problemas impulsa la cooperación.

Así que, si un Gobierno decide poner en marcha una política (digamos, generación de ingresos para víctimas) y ejecutarla al menos parcialmente mediante un programa interorganizacional con múltiples proyectos conjuntos entre ministerios y contrapartes locales (gobernaciones, alcaldías), pero la política no es completamente clara en lo que se debe hacer, los niveles de conflicto - entendido como restricción autoimpuesta a la cooperación- serán menores entre implementadores que comparten la misma idea de qué hacer y cómo hacerlo que si esos implementadores (los diferentes ministerios, alcaldías, gobernaciones) tienen ideas diferentes.

El postulado se representa gráficamente en la figura 3: 
FIGURA 3. RELACIÓN ENTRE ESTRUCTURACIÓN DEL PROBLEMA Y ÉXITO EN LA IMPLEMEMNTACIÓN

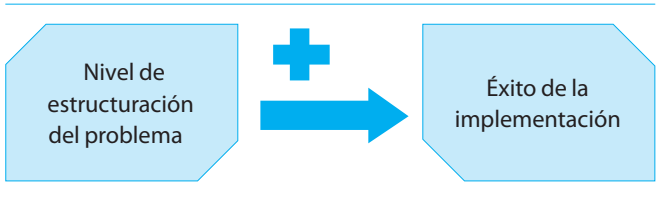

Fuente: elaboración propia.

\section{LAS HIPÓTESIS}

La afirmación general del modelo se concreta en tres hipótesis para las cuales utilizamos ya no los constructos generales (estructuración del problema, ambigüedad de política, implementación exitosa), sino las variables operacionales.

\section{Hipótesis 1}

Si el constructo teórico "estructuración del problema” es definido más precisamente en términos de dos variables operacionales (estructuración de problemas respecto a objetivos y estructuración de problemas respecto a medios), es posible predecir una dirección general de las relaciones entre esas variables y la variable interviniente conflicto, siempre que se esté en presencia de ambigüedad en la política. No nos referimos a correlaciones, primero, porque asumimos que una cuantificación precisa de las variables con las que tratamos simplemente no es posible, y, segundo, porque, aún si fuese posible, tantas variables podrían estar "escondidas" que no tendría sentido un ejercicio de atribución (que es normalmente el propósito de identificar correlaciones).
La aparición de conflicto estará asociada con situaciones en las que politicas/mandatos ambiguos sobre objetivos y medios coincidan con problemas no estructurados (no hay consenso sobre objetivos ni sobre medios). Pero también con situaciones en las que una de las dimensiones de la politica o el mandato (objetivos o medios) sea ambigua, y la misma dimensión del problema no esté estructurada entre los implementadores.

Si un Gobierno implementa un programa interorganizacional de generación de ingresos con múltiples proyectos conjuntos, tenderemos a encontrar conflicto en aquellos proyectos en los que el mandato no sea completamente claro sobre lo que se quiere (aumentar los ingresos en x o y porcentaje, hacerlo con o sin enfoque diferencial, etcétera) y coincida con un problema no estructurado en cuanto a eso mismo que se quiere (objetivos), o el mandato no sea claro sobre los medios susceptibles de considerar (proyectos individuales o colectivos, solo en zonas urbanas o también rurales), y coincida con un problema no estructurado en cuanto a medios. Y también habrá conflicto, por supuesto, cuando no haya claridad en el mandato ni sobre objetivos ni sobre medios, y el problema tampoco esté estructurado entre los implementadores sobre ninguna de estas dos dimensiones. La idea se representa gráficamente en la figura 4 .

\section{FIGURA 4. EL CONFLICTO COMO RESULTADO DE LA INTERFACE ENTRE ESTRUCTURACIÓN Y AMBIGÜEDAD}

(ESTRUCTURA PRO) $($ AMBIG POL) $=$

CONFLICTO

Fuente: elaboración propia. 


\section{Hipótesis 2}

Es posible predecir la dirección de las relaciones entre la presencia de restricciones autoimpuestas a la cooperación (conflicto) relacionadas con el problema, y las variables dependientes ritmo de implementación y variación (dentro de una misma intervención, sea programa, proyecto o norma). Aunque no es posible hablar de "correlaciones" dadas las dificultades esperadas de medición y la probable presencia de variables "ocultas" que no puedan ser controladas, es posible encontrar relaciones negativas entre conflicto y ritmo, y relaciones positivas entre conflicto y variación.

En el mismo ejemplo de implementación de un programa interorganizacional de generación de ingresos constituido por múltiples proyectos, asumiendo ambigüedad en las directrices del programa, el ritmo de implementación será más alto en aquellos casos en que las combinaciones de ambigüedad y estructuración del problema conduzcan a menor conflicto. $\mathrm{Y}$, al mismo tiempo, las variaciones en los proyectos (cambios en alcance, redefinición de actividades, etcétera) serán menores.

\section{Hipótesis 3}

Por último, la intensidad del efecto del conflicto sobre el ritmo y la variación en la implementación estará relacionada con el nivel de interdependencia entre los implementadores, siendo menor el ritmo y mayor la variación cuando el nivel de interdependencia requerido es más alto.

Es importante recordar que las categorías que usaremos para la interdependencia son aquellas creadas por Thompson (1967) y adoptadas luego por O’Toole (1984, 2007), identificando tres tipos de interdependencia: operacional combinada, operacional secuencial y operacional recíproca, nombradas aquí en orden creciente de interdependencia entre los implementadores.

Si volvemos al ejemplo de los proyectos de nuestro programa interorganizacional conjunto de generación de ingresos, los menores ritmos de implementación y las mayores variaciones podemos esperarlos en proyectos en que se produzca conflicto y se requiera interdependencia operativa recíproca, con niveles de afectación también altos, pero no tanto, en situaciones de interdependencia secuencial, y los efectos más bajos cuando el conflicto aparece en proyectos que solo requieren interdependencia operacional combinada. Las relaciones entre conflicto, interdependencia requerida, ritmo de implementación y variación podrían graficarse como se muestra en la figura 5 .

FIGURA 5. FALTA TÍTULO

$($ CONFLICTO) $($ INTERDEPENDENCIA REQUERIDA $)=($ RITMO, VARIACIÓN)

Fuente: elaboración propia.

¿Cómo resumir las implicaciones de las tres hipótesis? La figura 6 muestra ocho combinaciones diferentes de nivel de estructuración de problemas y ambigüedad de política, combinando cuatro posibles niveles de estructuración de problemas (problema estructurado PE; problema medianamente 
estructurado, estructurado respecto a medios $\mathrm{PE}(\mathrm{m})$; problema medianamente estructurado, estructurado respecto a objetivos PE(o); y problema no estructurado PNE), cada una con dos posibilidades de ambigüedad de política: ambigüedad respecto a medios y ambigüedad respecto a fines. En aras de la simplicidad, las situaciones donde hay ambigüedad de la política no son tratadas aquí, ya que nuestro modelo se ocupa precisamente del efecto de la ambigüedad de la política. Sin embargo, como advertimos arriba, en contextos interorganizacionales donde no se espera el ejercicio de la autoridad para garantizar la cooperación, es posible que el conflicto (restricción autoimpuesta de la cooperación por algunos participantes) se presente incluso sin que exista ambigüedad.

De las ocho diferentes combinaciones, el modelo anticipa conflicto en la mitad de ellas ( 1 y 2, cuando problemas no estructurados se encuentran con cualquier tipo de ambigüedad de política, 3 cuando problemas moderadamente estructurados respecto a medios se encuentran con ambigüedad de política respecto a objetivos, 4 cuando problemas moderadamente estructurados respecto a objetivos se encuentran con ambigüedad de política respecto a medios). El conflicto relacionado con problemas es anticipado en todas las circunstancias teóricas en que la dimensión no estructurada de un problema se encuentra con ambigüedad de política relacionada con esa misma dimensión.

Ahora, por cada una de esas ocho parejas entre las posibilidades de nivel de estructuración y ambigüedad de la política hay tres niveles de influencia sobre el ritmo y la variación, dependiendo del tipo de dependencia entre implementadores de que estemos hablando: influencia más bien ligera en casos donde la intervención demanda solo interdependencia operacional conjunta (IOC); una influencia moderada en casos en que la intervención requiera una interdependencia operativa secuencial (IOs) más fuerte; y una influencia máxima allí donde el esquema de la intervención es de interdependencia operativa recíproca (IOR).

Aquellas situaciones en que hay conflicto (una restricción autoimpuesta a la cooperación, que puede llegar a la disputa abierta que concibe Matland) pero el programa/proyecto/ norma no requiere mucha cooperación entre los implementadores, son el entorno donde los efectos positivos del conflicto a los que se refirió Matland florecen, ya que la innovación prospera con el menor dańo (teórico) para el ritmo de implementación.

El ritmo puede ser menor (RIME) cuando hay conflicto pero solo se requiere interdependencia operacional combinada (IOC), deficiente cuando hay conflicto y se requiere interdependencia operacional conjunta (IOC); deficiente (RIDEF) cuando hay conflicto y se requiere interdependencia operacional secuencial; y mínimo (RIMn) cuando el conflicto combina interdependencia operacional recíproca. En cuanto a la variación, esta sería baja pero incrementada (vARBaj+) para la combinación entre conflicto e interdependencia conjunta, media (VARMd) para la combinación entre conflicto e interdependencia secuencial, y máxima (VARMx) para los casos en los que el conflicto coincida con los máximos niveles de interdependencia. Cuando el conflicto está ausente, el ritmo debería ser mayor (RI+) y la 
variación baja para cualquier nivel de interdependencia, en general, controlando otras variables. Por las razones explicadas arriba, aquí solo podemos esperar identificar regularidades estadísticas, sin correlaciones precisas, dada la imposibilidad de la cuantificación precisa de las variables y las dificultades de controlar efectivamente todas ellas (figura 6).

\section{CONCLUSIONES}

La investigación de la implementación puede beneficiarse de una definición más precisa, y de explorar el rol de la estructuración del problema (entendida como consenso en las dimensiones de objetivos y medios) sobre ella.

\section{FIGURA 6. DIFERENTES COMBINACIONES DE ESTRUCTURACIÓN DEL PROBLEMA Y AMBIGÜEDAD DE LA POLÍTICA, EFECTOS ESPERADOS SOBRE EL RITMO DE IMPLEMENTACIÓN Y LA VARIACIÓN}
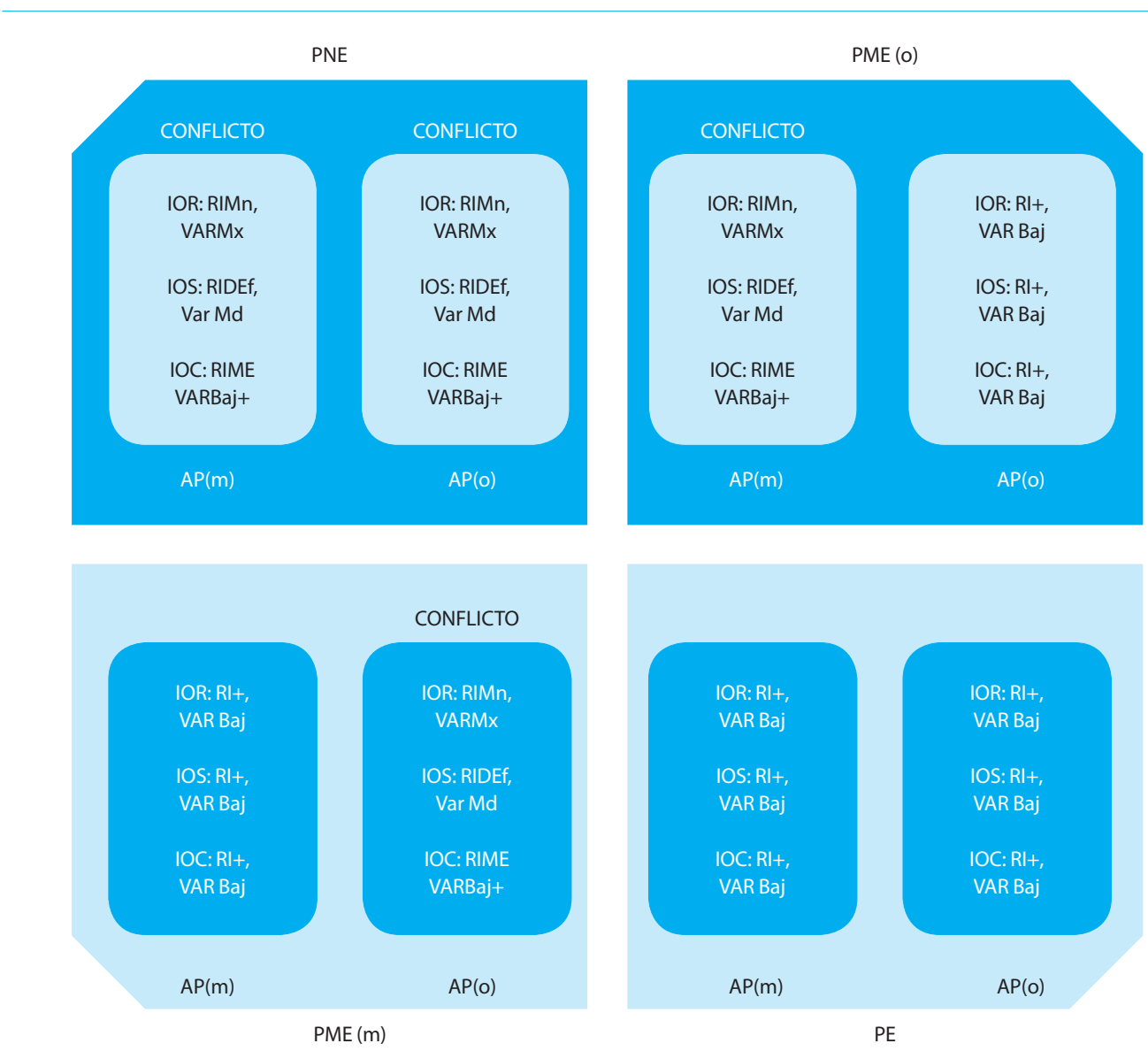

Fuente: elaboración propia. 
El constructivismo nos ofrece una salida al dilema de los "problemas irresolubles" explicando por qué son irresolubles, y la aproximación concreta de Robert Hoppe (2010) nos permite trabajar con dos dimensiones (objetivos y medios) comunes a los problemas, las alternativas reflejadas en políticas, programas y proyectos, y a la ya larga tradición de la literatura de análisis de políticas.

El propósito de este modelo es explicar por qué, concretamente, la estructuración del problema afecta la implementación - lo que logramos explicar, recurriendo al trabajo de Matland (1995) mediante el efecto de la estructuración sobre el conflicto-y por qué, como observaron O’Toole y Montjoy (1984), el conflicto no tiene siempre el mismo nivel de efectos negativos - lo que iluminamos mediante la explicación de los mismos O’Toole y Montjoy sobre el efecto de que existan distintos niveles de interdependencia en implementaciones interorganizacionales-.

El gran aporte que esperamos realizar a la investigación es el de someter a "falsificación" las tres hipótesis que concretan nuestro planteamiento general, y aumentar nuestro nivel de confianza en el planteamiento como resultado —o descubrir que ese planteamiento no es una explicación plausible, si es el caso-.

Si, utilizando el método popperiano, el modelo no se prueba errado, sus implicaciones serán particularmente relevantes en contextos de implementación interorganizacional, la misma que continúa expandiéndose también en Colombia. Algunos gobiernos crean "sistemas" de política para coordinar acciones de múltiples organizaciones gubernamentales respecto a problemas de política complejos. Y muchas veces la coordinación no está resolviendo los problemas de cooperación.

La administración pública no estará en capacidad de resolver las múltiples dificultades de la implementación de políticas, programas y proyectos con solo reconocer el papel de la estructuración de problemas en la ecuación, pero podrá administrar mejor el "riesgo" de problemas en la implementación tratando de evitar situaciones en que múltiples implementadores, sin una visión compartida de los objetivos y los medios, sean lanzados a implementar esas políticas, programas y proyectos.

No se han diseñado aún indicadores específicos para medir cada una de las variables operacionales señaladas arriba. Una exploración de las fuentes de información disponibles es necesaria para elegir esos indicadores. Identificar los casos adecuados por estudiar será el siguiente reto de este modelo.

\section{REFERENCIAS}

Ackoff, R. L. (1974). The social responsibility of operational research. Operational Research Quarterly. 25 (3), 361-371.

Bardach, E. (1977). The implementation game: what happens after a bill becomes a law. Cambridge: The MIT Press.

Birkland, T. A. (2011). An introduction to the policy process: theories, concepts and models of public policy making. New York: M.E. Sharpe, Inc.

Bouma, G. D. y Ling, R. (2004). The research process. South Melbourne: Oxford University Press.

Dunn, W. N. (2008). Public Policy analysis: an introduction. New Jersey: Pearson-Prentice Hall. 
Dunn, W. N. (1988). Methods of the second type: Coping with the wilderness of conventional policy analysis. Policy Studies Review, 7 (4), 720-737.

Dye, T. R. (1987). Understanding Public Policy. New Jersey: Prentice Hall.

Hill, M. y Hupe, P. (2007). Implementing public policy. London: Sage.

Howlett, M (2011). Designing public policies: principles and instruments. Oxon: Routledge.

Hoppe, R. (2010). The Governance of Problems: puzzling, powering and participation. Bristol: The Policy Press.

Jones, C. O. (1984). An introduction to the study of public policy. Monterrey: Brooks/Cole Publishing Company.

Lindblom, C. E. (1959). The Science of "Muddling Through”. Public Administration Review, 19 (2), 79-88.

Majone, G. (2005). Evidencia, argumentación y persuasión en la formulación de politicas. México, D.F: Fondo de Cultura Económica.

Matland, R. E. (1995). Synthesizing the implementation literature: the ambiguity-conflict model of policy implementation. Journal of Public Administration Research and Theory, 5 (2), 145-174.

May, P. (1993). Mandate design and implementation. Enhancing implementation efforts and shaping regulatory styles. Journal of Policy Analysis and Implementation, 12 (4), 634-663.

Mazmanian, D. A. y Sabatier, P. (1989).Implementation and public policy. Washington, D.C: University Press of America.

Mingers, J. y Rosenhead, J. (2009). A new paradigm for analysis. En Rosenhead, J. y Mingers, J. (eds.). Rational analysis for a problematic world revisited. Chichester (UK): John Wiley \& sons.

O’Toole, L. (2007). Interorganizational relations in implementation. En Peters, B. G. y Pierre, J. (eds.) Handbook of Public Administration. London: SAGE.

O’Toole, L. (2000). Research on public implementation: assessment and prospects. Journal of Public Administration Research and Theory, 10 (2), 263-288.

O’Toole, L. (1986). Policy recommendations for multiactor implementation: an assessment of the field. Journal of Public Policy, 6 (2), 181-210.

O’Toole, L. y Montjoy, R. S. (1984). Interorganizational policy implementation: A theoretical perspective. Public Administration Review, 44 (6), 491-503.

Pressman, J. y Wildavsky, A. (1973). Implementation. Berkeley: University of California Press.

Raiffa, H. (1968). Decision analysis. Reading: AddisonWesley.

Rittel, H. y Webber, M. (1973). Dilemmas in a General Theory of Planning. Policy Sciences 4, 155-169.

Sabatier, P. A. y Jenkins-Smith, H. (1993). Policy change and learning: an advocacy coalition approach. Boulder: Westview Press

Sandfordt, J., Moulton, S. y Roll, S. (2013). Integrating and advancing policy and program implementation analysis. Washington, D.C.: Documento presentado en la conferencia anual de investigación de la Association for Public Policy Analysis and Management.

Simon, H. (1973). The structure of ill-structured problems. Artificial intelligence (4), 181-201.

Thompson, J. D. (1982). Bureaucratic discretion and the National Health Service Corps. Political Science Quarterly, 97 (3), 427-445.

Thompson, J. D. (1967). Organizations in action. New York: McGraw-Hill.

Valdivieso, G. (2013). The problem of problem structuredness in the implementation research literature: a state of the art. Recuperado de: www. academia.edu 
van Meter, D. y van Horn, C. E. (1975). The policy implementation process: a conceptual framework. En Aguilar, L. F. (ed.) (1993). La implementación de las politicas. México, D.F.: Miguel Angel Porrúa.

Yin, R. (2009). Case study research: design and methods. Los Angeles: SAGE. 